\title{
Indicadores de desigualdad y mortalidad por enfermedades cardiometabólicas en Guatemala
}

\author{
Inequality indicators and cardiometabolic diseases mortality in Guatemala \\ Alejandro Cerón (D) *, Gila Y. Goldstein (D) \\ University of Denver, Colorado, Estados Unidos
}

*Autor al que se dirige la correspondencia: Alejandro.CeronValdes@du.edu

Recibido: 05 de julio 2001 / Revisión: 13 de julio 2021 / Aceptado: 28 de octubre 2021

\section{Resumen}

T a medición de las desigualdades en salud al interior de los países de ingresos bajos y medios es necesaria para Lla planificación, monitoreo y evaluación de intervenciones de salud pública, especialmente para problemas que contribuyen altamente a la carga de enfermedad, como las enfermedades cardiometabólicas. El objetivo de este estudio fue caracterizar los patrones de desigualdad de las tasas de mortalidad para las principales causas cardiometabólicas en Guatemala. Se usó datos del Censo Nacional de Población, y estadísticas oficiales de defunción de 2018 para calcular tasas crudas de mortalidad para diabetes (DM), infarto agudo de miocardio (IAM), y accidente cerebrovascular (ACV). Se calcularon indicadores simples y complejos de desigualdad absoluta y relativa (diferencia, razón, índice de pendiente, índice de concentración, distancia de la media, índice de Theil, riesgo atribuible poblacional, y porcentaje de riesgo atribuible poblacional) para seis dimensiones de desigualdad: sexo, pueblo de pertenencia, nivel educativo, tipo de ocupación, departamento y municipio. Se documentaron 6,445 muertes por DM, 5,761 por IAM, y 3,218 por ACV. Los indicadores mostraron marcadas desigualdades para las seis dimensiones estudiadas. El patrón de desigualdad predominante para sexo, pueblo de pertenencia y departamento fue de privación masiva. Para nivel educativo y ocupación predominó un patrón de incrementos escalonados. Se identificó exclusión marginal superpuesta en nivel educativo, ocupación y municipio. Se concluye que los patrones de desigualdad de las tasas de mortalidad para estas tres enfermedades sugieren la superposición de patrones de privación masiva, incrementos escalonados, y exclusión marginal.

Palabras claves: Desigualdades en la Salud, Enfermedades no Transmisibles, Epidemiología, Guatemala

\begin{abstract}
$\mathrm{M}$ easuring health inequalities within low- and middle-income countries is needed for planning, monitoring, and evaluation of public health interventions, especially for problems that represent a high burden of disease, like cardio metabolic diseases. The goal of this study was to characterize inequality patterns in mortality rates from cardio metabolic causes in Guatemala. Data from the 2018 National Population Census, and official vital statistics were used to estimate crude mortality rates for diabetes (DM), acute myocardial infarction (IAM), and stroke (ACV). Simple and complex indicators of absolute and relative inequality (difference, ratio, slope index, concentration index, distance from the mean, Theil index, population attributable risk, and percentage of population attributable risk) were calculated for six dimensions of inequality: sex, race/ethnicity, education level, type of occupation, department, and municipality. A total of 6,445 DM deaths, 5,761 IAM deaths, and 3,218 ACV deaths were documented. Indicators showed marked inequalities for the six dimensions studied. Massive deprivation was the predominant inequality pattern for sex, race/ethnicity, and department. Staggered increments were identified for education level and type of occupation. Overlapping marginal exclusion was found for education level, occupation, and municipality. We conclude that inequality patterns found for the three causes of death suggest overlapping patterns of mass deprivation, staggered increments, and marginal exclusion.
\end{abstract}

Keywords: Health Status Disparities, Noncommunicable Diseases, Epidemiology, Guatemala 


\section{Introducción}

La medición y monitoreo de las desigualdades en salud al interior de los países de ingresos bajos y medios es una necesidad a nivel mundial (Organización Panamericana de la Salud [OPS], 2018). La distinción conceptual entre desigualdades e inequidades en salud se generalizó en los años 1990 (Pan American Health Organization [PAHO], 1995), cuando también hubo desarrollos metodológicos para su medición (Anand et al., 2001; Braveman, 1998; Keppel et al., 2005; Wagstaff, 1991; Regidor, 2004a,2004b), los cuales recibieron cierta atención en las discusiones sobre los Objetivos de Desarrollo del Milenio, y un renovado auge con los Objetivos de Desarrollo Sostenible. La medición, análisis y monitoreo de las desigualdades en salud ha sido señalado como un pilar para el abordaje de las inequidades en salud (Commission on Social Determinants of Health [CSDH], 2008; World Health Organization [WHO], 2013, 2017), sin embargo las evidencias disponibles indican que esas mediciones, análisis y monitoreo son casi inexistentes en los países de ingresos bajos y medios debido al escaso financiamiento para la investigación, poca disponibilidad de datos, reducidas capacidades técnicas, y falta de prioridad política (Mújica \& Moreno, 2019). Los análisis empíricos recientes de las inequidades al interior de países de ingresos bajos y medios han aumentado recientemente (Enríquez Nava et al., 2020; Gutiérrez et al., 2016; Tullo et al., 2020).

Las enfermedades cardiometabólicas están entre las que más contribuyen a la carga mundial de enfermedad medida como años de vida perdidos por discapacidad (DALYs) y tienen un gran impacto en la calidad de vida de muchas poblaciones, así como en los recursos de los sistemas de salud (GBD 2019 Diseases and Injuries Collaborators, 2020). En Guatemala, la diabetes mellitus (DM), el infarto agudo de miocardio (IAM), y el accidente cerebrovascular (ACV) son unas de las principales causas de muerte (Institute for Health Metrics and Evaluation [IHME], 2020b). Las enfermedades cardiometabólicas comparten una serie de factores de riesgo como la hipertensión arterial, tabaquismo, hiperglicemia, obesidad, hipercolesterolemia, y falta de actividad física, entre otros (Mendis et al., 2011). Todos estos factores de riesgo van en franco aumento en Guatemala (Guzmán, 2013; Rodas Estrada et al., 2013; The WHO CVD Risk Chart Working Group 2019). Guatemala es uno de los países en los que la DM, el IAM y el ACV tienen un mayor impacto en DALYs y en mortalidad (Flores Ramírez, 2002; IHME, 2020a; Moscoso \& Flores, 2008). La mortalidad por DM se ha incrementado de 18 a 36 muertes por 100,000 habitantes entre 2001 y 2018 (Sam Colop, 2017, 2018). La mortalidad por IAM se ha incrementado desde 19 a 33 muertes por 100,000 habitantes entre 2008 y 2018, y la mortalidad por ACV ha ido de 11 a 21 muertes por 100,000 habitantes en el mismo período (Sam Colop, 2017, 2018). Análisis descriptivos de las tasas de mortalidad para estas enfermedades sugieren patrones de desigualdad en múltiples dimensiones (Cerón \& Goldstein 2021a, 2021b, 2021c).

El objetivo de este estudio fue caracterizar los patrones de desigualdad de las tasas de mortalidad para las principales causas cardiometabólicas en Guatemala. Se usó datos del Censo Nacional de Población (Instituto Nacional de Estadística [INE], 2020), y estadísticas oficiales de defunción (INE, 2019) de 2018 para calcular tasas crudas de mortalidad para diabetes (DM), infarto agudo de miocardio (IAM), y accidente cerebrovascular (ACV). Se calcularon indicadores simples y complejos de desigualdad absoluta y relativa para seis dimensiones de desigualdad. Los indicadores mostraron marcadas desigualdades para las seis dimensiones estudiadas, mostrando distintos patrones para las diferentes dimensiones de desigualdad estudiados, que sugieren la superposición de patrones de privación masiva, incrementos escalonados, y exclusión marginal. Los resultados de este estudio ofrecen evidencias empíricas que pueden llevar a una teorización de las desigualdades en salud que oriente intervenciones intencionadas de acuerdo a los distintos patrones de desigualdad identificados.

\section{Materiales y métodos}

Se hizo análisis secundario de bases de datos del Instituto Nacional de Estadística de Guatemala (INE). Se calculó tasas crudas de mortalidad para las tres enfermedades. Para los numeradores se usó la base de datos de defunciones anuales de 2018 (INE, 2019) utilizando las definiciones operativas utilizadas por el proyecto de la Carga Global de Enfermedades (GBD 2019 Diseases and Injuries Collaborators, 2020), que comprende los siguientes códigos de la $10^{\mathrm{a}}$ edición de los Códigos Internacionales de Enfermedades (CIE-10). Para diabetes mellitus (DM), E08-E08.11, E08.3-E08.9, E10-E10.11, E10.3-E11.1, E11.3-E12.1, E12.3-E13.11, E13.3-E14.1, E14.3-E14.9, R73-R73.9, Z13.1, Z83.3. Para infarto agudo de miocardio (IAM), I20-I21.6, I21.9-I25.9, Z82.4-Z82.49. Para accidente 
cerebrovascular (ACV), G45-G46.8, I60-I62, I62.9-I64, I64.1, I65-I69.998, Z82.3. Para los denominadores se usaron las poblaciones obtenidas en el XII Censo Nacional de Población y VII de Vivienda de 2018 (INE, 2020).

Las dimensiones de desigualdad utilizadas son las disponibles en la base de datos de defunciones del INE, provenientes de los certificados oficiales de defunción: sexo, pueblo, nivel educativo, tipo de ocupación, departamento, y municipio. La dimensión sexo está clasificada como masculino o femenino. La dimensión pueblo, entendida como el grupo de adscripción basado en características de pertenencia cul- tural, lingüística o étnica, está clasificada como maya, garífuna, xinka, o mestizo/ladino. La dimensión nivel educativo está clasificada en las categorías presentadas en la Tabla 1. La dimensión tipo de ocupación utiliza la Clasificación Internacional Uniforme de Ocupaciones de la Organización Internacional del Trabajo de 2008, que comprende las categorías presentadas en la Tabla 2. Finalmente, se usaron las divisiones políticoadministrativas oficiales de Guatemala, que está dividida en 22 departamentos y en 340 municipios.

Los indicadores de desigualdad se definieron siguiendo las recomendaciones de la Organización

Tabla 1

Tasas crudas de mortalidad por 100,000 habitantes por diabetes, infarto agudo de miocardio y accidente cerebrovascular por sexo, pueblo y nivel educativo. Guatemala 2018

\begin{tabular}{lccc}
\hline & Diabetes & Infarto agudo de miocardio & Accidente cerebrovascular \\
\hline $\begin{array}{l}\text { Por sexo } \\
\text { Femenino }\end{array}$ & 51 & 35 & 21 \\
Masculino & 35 & 43 & 22 \\
Por pueblo & & & \\
Maya & 29 & 19 & 13 \\
Garífuna & 26 & 36 & 10 \\
Xinka & 0 & 3 & 0 \\
Mestiza o ladina & 50 & 49 & 25 \\
Por nivel educativo & & 110 & 65 \\
Sin escolaridad & 110 & 87 & 48 \\
Primaria & 40 & 11 & 6 \\
Secundaria & 14 & 21 & 9 \\
Bachillerato & 24 & 21 & 9 \\
Licenciatura & 21 & 59 & 22 \\
Postgrado & 5 & 39 & \\
Población total & 43 & & \\
\hline & & 5 & \\
\hline
\end{tabular}


Mundial de la Salud, OMS (WHO, 2013, 2017). Los indicadores simples de desigualdad incluyen la diferencia y la razón. Para la diferencia se restó la tasa de mortalidad más alta menos la más baja, para cada una de las dimensiones de desigualdad. Para la razón se dividió la tasa de mortalidad más alta entre la más baja, para cada una de las dimensiones de desigualdad. El índice de pendiente y el índice de concentración son dos indicadores complejos de desigualdad que pueden usarse sólo con dimensiones de desigualdad con clasificaciones ordinales, como el nivel educativo y el tipo de ocupación. El índice de pendiente, por ser un indicador de desigualdad absoluta, está expresado en muertes por 100,000 habitantes, donde valores absolutos más altos muestran mayor desigualdad y el signo negativo muestra que las muertes están más concentradas entre la población con niveles educativos más bajos u ocupaciones de menos jerarquía. El índice de concentración, por ser un indicador de desigualdad relativa, es adimensional, y fluctúa entre valores absolutos teóricos de 0 para la máxima igualdad y 1 para la máxima desigualdad, y el signo negativo indica concentración entre los grupos de menos jerarquía. La distancia de la media y el índice de Theil son dos indicadores complejos que pueden usarse sin importar si la dimensión de desigualdad está clasificada de manera ordinal o no. La distancia de la media, al ser una medida absoluta de desigualdad, se expresa en muertes por 100,000 habitantes, donde valores más altos muestran mayor desigualdad. El índice de Theil, por ser un indicador relativo, es adimensional, no puede tener valores con signo negativo, y fluctúa entre el 0 de la total igualdad y el infinito, por lo que valores más altos indican mayor desigualdad. El riesgo atribuible poblacional muestra el margen de mejora si las desigualdades en el indicador se eliminaran y todos los grupos mostraran tasas de mortalidad iguales a la del subgrupo con menor mortalidad. Todo el procesamiento de datos y cálculo de indicadores se hizo con Microsoft Excel.

\section{Resultados}

Se documentó un total de 6,445 muertes por diabetes mellitus (DM), de 5,761 muertes por infarto agudo de miocardio (IAM), y de 3,218 muertes por accidente cerebrovascular (ACV). Descripciones detalladas de la distribución de las tasas de mortalidad se han publicado (Cerón \& Goldstein 2021a, 2021b, 2021c), y se presenta una síntesis en las Tablas 1 y 2. Una comparación entre subgrupos dentro de las diferentes dimensiones de desigualdad sugiere que hay desigualdades en la distribución de las tasas de mor-

Tabla 2

Tasas crudas de mortalidad por 100,000 habitantes por diabetes, infarto de miocardio y accidente cerebrovascular por ocupación. Guatemala 2018

\begin{tabular}{lccc}
\hline Categoría de ocupaciones & Diabetes & Infarto agudo de miocardio & Accidente cerebrovascular \\
\hline Militares & 0 & 24 & 12 \\
Directivas y gerenciales & 11 & 4 & 3 \\
Científicas e intelectuales & 48 & 42 & 18 \\
Técnicas de nivel medio & 51 & 36 & 14 \\
De asistencia administrative & 26 & 21 & 11 \\
Servicios y vendedores & 46 & 29 & 15 \\
Agropecuarias calificadas & 6 & 10 & 7 \\
Operarios y artesanos & 47 & 40 & 19 \\
Maquinistas y ensambladores & 62 & 42 & 17 \\
Ocupaciones elementales & 81 & 129 & 69 \\
Ocupaciones no clasificadas & 40 & 30 & 16 \\
Población total & 43 & 39 & 22 \\
\hline
\end{tabular}


talidad. Los indicadores de desigualdad para las tasas de mortalidad por DM, IAM, y ACV se presentan en las Tablas 3, 4 y 5, respectivamente.

Para DM (Tabla 3), la diferencia de muertes por 100,000 habitantes para las diferentes dimensiones de desigualdad va desde las 16 muertes más en mujeres, hasta las 105 muertes más en población sin escolaridad comparada con quienes tienen estudios de postgrado. La razón muestra en un extremo que hubo 1.5 muertes de mujeres por cada muerte de hombres, y en el otro extremo que hubo 22 muertes en población sin escolaridad por cada muerte entre quienes tienen estudios de postgrado. Los dos índices de pendiente son negativos, mientras que los dos índices de concentración son cercanos a cero. La distancia de la media más alta se da entre municipios, seguida por pueblos, y ocupación, mientras que la más baja se da en sexo y departamentos. Por su parte, el índice de Theil es marcadamente más alto para pueblo, nivel educativo y municipios. El riesgo atribuible poblacional y su porcentaje muestran valores más altos para municipio, educación y ocupación.

Para IAM (Tabla 4), la diferencia va de 8 a 125 muertes por 100,000 habitantes para sexo y ocupación, respectivamente. Las diferencias para departamento y municipio son mucho más altas que las de DM. La razón más alta es por ocupación, seguida de cerca por educación y departamento. Los índices de pendiente son negativos y más pronunciados que los de DM. Los índices de concentración son cercanos a cero. Las distancias de la media más altas se dan por municipio y ocupación, seguidos por pueblo. Los índices de Theil son más altos que los de DM, especialmente para educación, ocupación y municipio. El riesgo atribuible poblacional y su porcentaje son similares a los de DM, aunque es marcadamente menor para sexo y mayor para pueblo y departamento.

Para ACV (Tabla 5), la diferencia va de 1 para sexo a 66 para ocupación, seguido de cerca por nivel educativo. La razón va de 1 para sexo a 32 para nivel educativo, seguido de cerca por ocupación. Los índices de pendiente son negativos y menos pronunciados que los de DM. Los índices de concentración son cercanos a cero. Las distancias de la media son menores a las de DM e IAM, con las más altas para municipios y ocupación. Los índices de Theil son más altos que los de DM e IAM para ocupación, nivel educativo, y pueblo, además de que los de departamento y municipio son

Tabla 3

Indicadores de desigualdad para mortalidad por diabetes. Guatemala 2018

\begin{tabular}{lcccccc}
\hline \multirow{2}{*}{ Indicadores de desigualdad } & \multicolumn{5}{c}{ Dimensiones de desigualdad } \\
\cline { 2 - 7 } & Sexo & Pueblo & Educación & Ocupación & Departamento & Municipio \\
\hline Diferencia & 16 & 21 & 105 & 70 & 42 & 55 \\
Razón & 1.5 & 1.7 & 22.0 & 7.5 & 2.8 & NA \\
Índice de pendiente & $\mathrm{NA}$ & $\mathrm{NA}$ & -99.9 & -46.5 & $\mathrm{NA}$ & $\mathrm{NA}$ \\
Índice de concentración & $\mathrm{NA}$ & $\mathrm{NA}$ & -0.19 & 0.04 & $\mathrm{NA}$ & $\mathrm{NA}$ \\
Distancia de la media & 0.0 & 13.4 & 7.3 & 9.8 & 3.3 & 22.2 \\
Índice de Theil & 23.1 & 736.4 & 698.8 & 379.3 & 153.9 & 580.1 \\
Riesgo atribuible poblacional & 8.0 & 7.0 & 38.0 & 32.5 & 19.3 & 43.0 \\
Porcentaje de riesgo atribuible & 18.7 & 16.3 & 88.4 & 75.1 & 44.6 & 100.0 \\
poblacional & & & & & & \\
\hline
\end{tabular}

Nota. NA: No aplica 
Tabla 4

Indicadores de desigualdad para mortalidad por infarto agudo de miocardio. Guatemala 2018

\begin{tabular}{lcccccc}
\hline & \multicolumn{5}{c}{ Dimensiones de desigualdad } \\
\cline { 2 - 7 } Indicadores de desigualdad & Sexo & Pueblo & Educación & Ocupación & Departamento & Municipio \\
\cline { 2 - 7 } Diferencia & 8 & 30 & 105 & 125 & 105 & 107 \\
Razón & 1.25 & 2.6 & 22.0 & 32.2 & 18.5 & NA \\
Índice de pendiente & $\mathrm{NA}$ & $\mathrm{NA}$ & -120.05 & -81.7 & $\mathrm{NA}$ & NA \\
Índice de concentración & $\mathrm{NA}$ & $\mathrm{NA}$ & -0.20 & -0.09 & NA & NA \\
$\begin{array}{l}\text { Distancia de la media } \\
\text { Índice de Theil }\end{array}$ & 0.0 & 9.8 & 3.5 & 19.8 & 1.6 & 38.6 \\
$\begin{array}{l}\text { Riesgo atribuible poblacional } \\
\text { Porcentaje de riesgo atribuible }\end{array}$ & 2.1 & 518.2 & 1077.3 & 986.9 & 82.2 & 919.4 \\
poblacional & 4.0 & 20.0 & 34.0 & 35.0 & 33.0 & 39.0 \\
\hline
\end{tabular}

Nota. NA: No aplica

Tabla 5

Indicadores de desigualdad para mortalidad por accidente cerebrovascular. Guatemala 2018

\begin{tabular}{|c|c|c|c|c|c|c|}
\hline \multirow{2}{*}{ Indicadores de desigualdad } & \multicolumn{6}{|c|}{ Dimensiones de desigualdad } \\
\hline & Sexo & Pueblo & Educación & Ocupación & Departamento & Municipio \\
\hline Diferencia & 1 & 12 & 63 & 66 & 44 & 39 \\
\hline Razón & 1.0 & 1.9 & 32.0 & 23.0 & 9.8 & NA \\
\hline Índice de pendiente & NA & NA & -72.7 & -43.9 & NA & NA \\
\hline Índice de concentración & NA & NA & -0.26 & -0.12 & NA & NA \\
\hline Distancia de la media & 0.5 & 8.0 & 1.2 & 13.2 & 0.4 & 18.2 \\
\hline Índice de Theil & 0.4 & 896.2 & 1186.0 & 1160.5 & 81.7 & 880.4 \\
\hline Riesgo atribuible poblacional & 0.0 & 12.0 & 20.0 & 19.0 & 17.0 & 22.0 \\
\hline $\begin{array}{l}\text { Porcentaje de riesgo atribuible } \\
\text { poblacional }\end{array}$ & 0.0 & 54.5 & 90.9 & 86.4 & 77.3 & 100.0 \\
\hline
\end{tabular}

Nota. NA: No aplica 
casi tan altos como los de IAM. El riesgo atribuible poblacional y su porcentaje son similares a los de DM, aunque es marcadamente menor para sexo y mayor para pueblo.

\section{Discusión}

Los patrones de desigualdad de las tasas de mortalidad muestran características distintivas para diferentes dimensiones de desigualdad en las tres enfermedades analizadas. Partiendo de que las tasas de mortalidad para estas tres enfermedades es alta, las múltiples dimensiones de desigualdad sugieren la convivencia de patrones de privación masiva, incrementos escalonados, y exclusión marginal (WHO, 2013). La dimensión sexo muestra los menores niveles de desigualdad, aunque las mujeres tienen mayor probabilidad de morir de DM y los hombres de morir de IAM, lo que interpretamos como una privación masiva de las medidas de prevención primaria, secundaria y terciaria. La dimensión pueblo muestra mayores desigualdades en algunos indicadores, con la población mestiza siendo más afectada por las tres enfermedades, lo cual sugiere una privación masiva acentuada por exclusión marginal. Las dimensiones nivel educativo y ocupación presentan los indicadores más altos de desigualdad, los cuales presentan un patrón escalonado con tasas de mortalidad decrecientes conforme se sube en las jerarquías educativas y ocupacionales, pero que muestran diferencias muy marcadas en los niveles bajos de ambas jerarquías, lo que sugiere un patrón de exclusión marginal agregado. La distribución por departamentos sugiere un patrón de privación generalizada, mientras que la distribución por municipios sugiere un patrón de exclusión marginal.

La multidimensionalidad que muestran estos patrones de desigualdad tiene implicaciones para la investigación y práctica de la salud pública. En primer lugar, plantea la necesidad de entender las desigualdades desde la interseccionalidad, es decir entender las maneras como se intersectan las múltiples dimensiones de manera concreta en contextos concretos (Green et al., 2017; OPS, 2018). En segundo lugar, refuerza el llamado a comprender las condiciones de vida como el contexto inmediato en el que las desigualdades sociales generan inequidades en salud, así como a explicar los procesos sociales y macrosociales que generan dichas condiciones de vida (Breilh, 2021; CSDH, 2018; Krieger, 2011; OPS, 2018). Finalmente, invita a diseñar intervenciones de salud pública orientadas a la promoción de la salud, así como a la prevención primaria, secundaria y terciara, que toman en cuenta las múltiples dimensiones de las desigualdades en salud (Braveman, 1998; CSDH, 2018; Mújica \& Moreno, 2019; OPS, 2018).

En cuanto a las limitaciones de este estudio, es importante tomar en cuenta que los análisis que vienen de bases de datos de estadísticas vitales cuentan con limitaciones en la calidad de la definición de las variables y en la consistencia con que éstas son interpretadas por quienes generan los datos. Asimismo, no debe interpretarse que las dimensiones de desigualdad estudiadas son la causa de las diferencias en mortalidad, sino como patrones cuyas causas, condicionantes y determinantes deben ser investigados. La fortaleza del estudio es que se basa en estadísticas vitales que abarcan a toda la población y se generan de manera periódica, así como en datos censales de todo el país. Vale le pena considerar que la calidad de los datos generados por el sistema de estadísticas vitales de Guatemala ha sido evaluada recientemente como buena (WHO, 2019), y como "cuatro estrellas" de cinco posibles (GBD 2019 Diseases and Injuries Collaborators, 2020).

Los patrones de desigualdad de las tasas de mortalidad para estas tres enfermedades sugieren la convivencia de patrones de privación masiva, incrementos escalonados, y exclusión marginal de las medidas de promoción de la salud y prevención primaria, secundaria y terciaria de la enfermedad.

Para profundizar en la caracterización de las desigualdades en las enfermedades cardiometabólicas, se recomienda complementar el análisis con datos de morbilidad y mortalidad provenientes del sistema de información epidemiológica oficial, así como con estudios específicos con enfoque de riesgo, estudios que incorporen una perspectiva interseccional a las desigualdades, y estudios que profundicen en la determinación social de los patrones de desigualdad encontrados.

Para avanzar en la utilización de los indicadores de desigualdad para tasas de mortalidad, se recomienda establecer comparaciones con las mismas enfermedades en otros países, con otras enfermedades en el mismo país, y con otros momentos en el tiempo para las mismas enfermedades en el mismo país.

\section{Contribución de los autores}

Coordinación, elaboración y revisión del Documento: Todos los autores Diseño de la recolección de datos o del trabajo en campo: Todos los autores 
Recolección o contribución de datos o realización del trabajo de campo: Todos los autores

Limpieza, sistematización, análisis o visualización de datos: Todos los autores

Participación en análisis de datos, estructura y en la escritura del documento: Todos los autores

\section{Materiales suplementarios}

No tiene materiales suplementarios

\section{Referencias}

Anand, S., Diderichsen, F., Evans, T., Shkolnikov, V., \& Wirth, M. (2001). Measuring disparities in health: Methods and indicators. En T. Evans, M. Whitehead, F. Diderichsen, A. Bhuiya, \& M. Wirth, (Eds.), Challenging inequities in health: From ethics to action (pp. 48-67). Oxford University Press.

Braveman, P. (1998). Monitoring equity in health: A policy-oriented approach in low- and middleincome countries. World Health Organization.

Breilh, J. (2021). Critical Epidemiology and the People's Health: Theory and Context. Oxford University Press.

Cerón, A., \& Goldstein, G. (2021a). Mortalidad por accidente cerebrovascular en Guatemala 2018: Patrones e inequidades. Análisis de la Realidad Nacional, 10(203), 66-88.

Cerón, A., \& Goldstein, G. (2021b). Mortalidad por diabetes en Guatemala 2018: Patrones e inequidades. Análisis de la Realidad Nacional, 10(198), 76-98.

Cerón, A., \& Goldstein G. (2021c). Mortalidad por infarto agudo de miocardio en Guatemala 2018: patrones e inequidades. Análisis de la Realidad Nacional, 10(201), 76-97.

Commission on Social Determinants of Health. (2008). Closing the gap in a generation: Health equity through action on the social determinants of health: Final report of the commission on social determinants of health. World Health Organization.

Enríquez Nava, M. F., Esquivel Velásquez, A. D., Patón Sanjines, M., Pooley Ayarza, B. C., Alarcón, R., Hernández Muñoz, R., \& Sanhueza, A. (2020).
Comportamiento y desigualdades sociales en indicadores priorizados del Objetivo de Desarrollo Sostenible 3 en Bolivia. Revista Panamericana de Salud Pública, 44, Artículo e101. https://doi.org/10.26633/RPSP.2020.101

Flores Ramírez, C. (2002). Análisis y tendencias de la mortalidad en Guatemala, 1986 a 1999. . Ministerio de Salud Pública y Asistencia Social. GBD 2019 Diseases and Injuries Collaborators. (2020). Global burden of 369 diseases and injuries in 204 countries and territories, 1990-2019: A systematic analysis for the Global Burden of Disease Study 2019. Lancet, 396(10258),1204-1222. https://doi.org/10.1016/S0140-6736(20)30925-9

Green, M. A., Evans, C. R., \& Subramanian, S.V. (2017). Can intersectionality theory enrich population health research? Social Science and Medicine, 178, 214-216. https://doi.org/10.1016/j. socscimed.2017.02.029

Gutiérrez, J. P., García-Saisó, S., Espinosa-de la Peña, R., \& Balandrán, D. A. (2016). Desigualdad en indicadores de enfermedades crónicas y su atención en adultos en México: Análisis de tres encuestas de salud. Salud Pública de México, 58(6), 666-675. https://doi.org/10.21149/spm. v58i6.7923

Guzmán, I. (2013). Estado actual de los factores de riesgo cardiovascular en población general en Guatemala. Revista Medicina Interna, 17 (Suppl 1), s3-s7.

Institute for Health Metrics and Evaluation. (2020a). Global Burden of Disease summaries. Institute for Health Metrics and Evaluation, University of Washington. http://www.healthdata.org/results/ gbd_summaries/2019/

Institute for Health Metrics and Evaluation. (2020b). Guatemala. En Country profiles. Institute for Health Metrics and Evaluation, University of Washington. http://www.healthdata.org/guatemala Instituto Nacional de Estadística. (2019). Base de datos de defunciones en Guatemala, año 2018. https://www.ine.gob.gt/ine/vitales

Instituto Nacional de Estadística. (2020). Base de datos del XII Censo Nacional de Población y VII de Vivienda 2018. http://redatam. censopoblacion.gt/bingtm/RpWebEngine.exe/ Portal?BASE $=$ CPVGT2018\&lang $=$ esp 
Keppel, K., Pamuk, E., Lynch, J., Carter-Pokras, O., Insun, K., Mays, V., Pearcy, J., Schoenbach, V., \& Weissman, J. S. (2005). Methodological issues in measuring health disparities. Vital and Health Statistics, (141), 1-16.

Krieger, N. (2011). Epidemiology and the People's Health: Theory and Context. Oxford University Press.

Mendis, S., Puska, P., \& Norrving, B. (2011). Global Atlas on cardiovascular disease prevention and control.World Health Organization, World Heart Federation, and World Stroke Organization. https:// apps.who.int/iris/rest/bitstreams/53236/retrieve

Moscoso, V., \& Flores, C. (2008). Retratos de muertes evitables. El Sistema de Salud en Guatemala, ¿hacia dónde vamos? Programa de las Naciones Unidas para el Desarrollo. https://desarrollohumano. org.gt/biblioteca/serie-sobre-salud/

Mújica, O. J., \& Moreno, C. M. (2019). De la retórica a la acción: Medir desigualdades en salud para "no dejar a nadie atrás". Revista Panamericana de Salud Pública, (43), Articulo e12. https://doi.org/10.26633/RPSP.2019.12

Organización Panamericana de la Salud. (2018). Sociedades justas, equidad en salud y vidas dignas. Resumen ejecutivo del informe final de la Comisión OPS sobre equidad y desigualdades sociales en salud en las américas.

Pan American Health Organization. (1995). The search for equity: Annual report of the Director.

Regidor, E. (2004a). Measures of health inequalities: Part 1. Journal of Epidemiology and Community Health, 58(10), 858-861. https://doi.org/10.1136/ jech.2003.015347

Regidor, E. (2004b). Measures of health inequalities: Part 2. Journal of Epidemiology and Community Health, 58(11), 900-903. https://doi.org/10.1136/ jech.2004.023036

Rodas Estrada, M. A., Rodas Díaz, M. A., Montenegro, P., Corona, V., Sánchez, C., Rodas, A., Escaler, B., Wyss, F., \& Guzmán Melgar, I. (2013). Síndrome coronario agudo en Guatemala / Accute coronary syndrome in Guatemala. Revista Medicina Interna, 17(Suppl 1), s35-s42.
Sam Colop, B. (2017). Sala de Situación: Enfermedades no transmisibles 2017. Ministerio de Salud Pública y Asistencia Social, Departamento de Epidemiología. http://epidemiologia.mspas.gob.gt/ files/Publicaciones $\% 202018 /$ Enfermedades $\% 20$ No\%20Transmisibles/Analisis\%20anual\%20 ENT\%202017.pdf

Sam Colop, B. (2018). Sala de Situación: Enfermedades no transmisibles 2018. Guatemala: Ministerio de Salud Pública y Asistencia Social, Departamento de Epidemiología. http://epidemiologia.mspas.gob.gt/ files/Publicaciones $\% 202018 /$ Enfermedades $\% 20$ No\%20Transmisibles/Analisis\%20anual\%20 ENT\%202018.pdf

The WHO CVD Risk Chart Working Group. (2019). World Health Organization cardiovascular disease risk charts: Revised models to estimate risk in 21 global regions. Lancet Global Health, 7(10), Artículo E1332-E1345. https://doi.org/ 10.1016/S2214-109X(19)30318-3

Tullo, E., Lerea, M. J., González, R., Galeano, J., Insfrán, M. D., Muñoz, M., Aragón, M., \& Sanhueza, A. (2020). Desigualdades sanitarias y sociales en la salud materna y del niño en Paraguay. Revista Panamericana de Salud Pública, (44), Artículo e107. https://doi.org/10.26633/RPSP.2020.107

Wagstaff, A., Paci, P., \& Doorslaer, E. V. (1991). On the measurement of inequalities in health. Social Science and Medicine, 33(5), 545-557. https://doi.org/10.1016/0277-9536(91)90212-U

World Health Organization. (2013). Handbook on health inequality monitoring: With a special focus on low-and middle-income countries.

World Health Organization. (2017). National health inequality monitoring: A step-by-step manual.

World Health Organization. (2019). World Health Statistics 2019: Monitoring health for the SDGs. https://www.who.int/gho/publications/world health_statistics/2019/en/ 